\title{
Occlusal Adjustments in Implants and Natural Dentition - 3D Occlusion
}

Author: Vicente Jiménez-López

Publisher: Quintessence Publishing

Language: English

ISBN: 978-1-85097-292-1

Edition: 1/e

Publish Year: 2016

Pages: 240, illustrated

Price: $148.00 €$

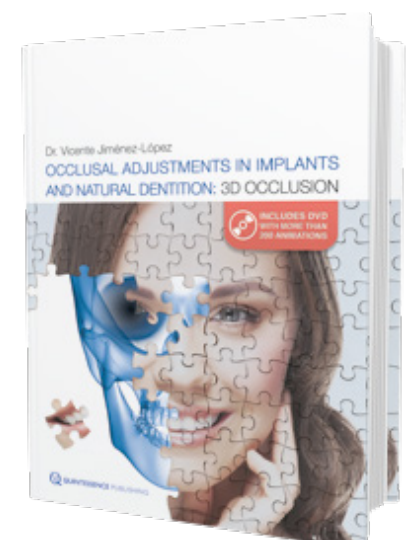

Occlusion is a very important part of maintaining the health of the stomatognathic system. Over time there have been many books on this subject, but Dr Vicente Jiménez-López manages to make o very compelling demonstration of this topic in his book entitled "Occlusal Adjustments in Implants and Natural Dentition - 3D Occlusion".

Starting from the operating philosophy that the human body needs to be evaluated from a holistic perspective for its proper functioning, it must provide the structures with a perfect balance in correlation with functional dental occlusion. After defining and illustrating the occlusion related terminology, the book presents the basic principles of organic occlusion. For any rehabilitation case it is essential that the quality of anterior guidance is evaluated first, continuing with centric relation, bilateral posterior occlusal stability and vertical dimension. These aspects are eloquently presented and illustrated in different chapters. The description and explanation of the prematurities and interferences that are the cause of the occlusal dysfunction and TMJ pathology are the outstanding achievements of this book. Completion of any oral rehabilitation case in implants and natural dentition requires occlusal adjustment in a stomatognathic system with relaxed muscles through the splint therapy. This book comes with an illustrative DVD which includes more than 200 clinical animations.

DrVicente Jiménez-López'book is a comprehensive presentation starting from the principles of occlusion and addressed both beginner and advanced dentists and technicians in practicing oral rehabilitation.

DOI: 10.25241/stomaeduj.2017.4(4).bookreview.1 